\title{
Anorectal melanoma
}

\author{
R.P. Jalleh, ${ }^{1 *}$ R. Pathmanathan, ${ }^{2}$ M.M.S. Krishnan ${ }^{1}$ and A. Mukherjee ${ }^{1}$ \\ Departments of ${ }^{1}$ Surgery and ${ }^{2}$ Pathology, University of Malaya, 59100 Kuala Lumpur, Malaysia.
}

\begin{abstract}
Summary: Four cases of anorectal melanoma are presented. The authors believe that this is the first report of the occurrence of this tumour in Malays. Advanced disease at initial presentation accounts for the poor prognosis observed in this series. Surgery remains the principal treatment modality, although controversy exists regarding optimal extent of resection.
\end{abstract}

\section{Introduction}

Anorectal melanoma is a rare malignant tumour associated with late detection and a poor prognosis. ${ }^{1}$ Since the initial description by Moore in $1857,{ }^{2}$ about 460 cases have been reported. ${ }^{3}$ Although the disease is rare in non-Caucasians, ${ }^{4,5}$ there have been isolated reports from the Asian region of occurrence in Indians ${ }^{6}$ and Chinese $^{7}$ patients. This report of four cases represents the initial Malaysian experience with this clinical rarity.

\section{Case reports}

\section{Case 1}

A 53 year old Malay man was admitted for anal pain 4 days after a banding procedure for haemorrhoids was performed. Rectal examination revealed a large circumferential pigmented rectal mass, the upper border of which was beyond the reach of the index finger. Biopsy confirmed the diagnosis of malignant melanoma. There was no inguinal lymphadenopathy or hepatomegaly. An emergency abdominoperineal resection was performed when the mass lesion prolapsed (Figure 1). At laparotomy, lymph nodes along the inferior mesenteric vessels were found to be enlarged and histopathology of these confirmed malignant involvement. Six months later, the patient was readmitted with right hemiplegia. Computerized tomography of the brain showed evidence of multiple metastatic nodules in the left cerebral hemisphere. He was discharged home with steroids for palliation.

Correspondence and *present address: R. Jalleh, F.R.C.S.(Ed), F.R.C.S.(Gks), F.R.C.S.I., Department of Surgery, Royal Gwent Hospital, Newport, Gwent NP7 2UB, UK

Accepted: 8 March 1988

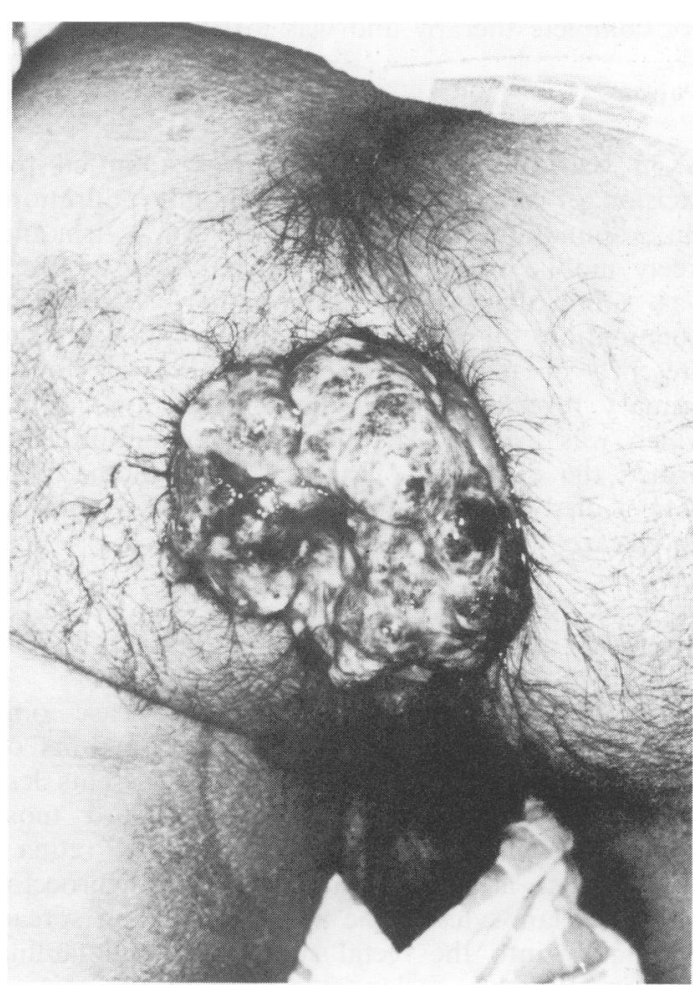

Figure 1 Prolapsed anorectal melanoma showing pigmentation.

Case 2

A 69 year old Indian man was admitted for jaundice and bleeding per rectum on defaecation. Abdominal examination was essentially normal. A firm, $3 \mathrm{~cm}$, pigmented and ulcerated lesion was noted in

(C) The Fellowship of Postgraduate Medicine, 1988 
the left wall of the anus. Biopsy of the mass showed the lesion to be a malignant melanoma. The cause of the jaundice was established to be hepatatis B virus hepatitis. At laparotomy, lymph nodes at the origin of the inferior mesenteric vessels were found to contain tumour. The patient succumbed 9 months after abdominoperineal resection.

\section{Case 3}

A 66 year old Malay woman presented with an anal mass of 2 months' duration. She was anaemic and rectal examination revealed a large fungating, circumferential mass. There was bilateral inguinal lymphadenopathy. Chest X-rays and liver function tests were normal. A diverting sigmoid colostomy was performed and the patient was subjected to palliative radiotherapy, with a dose of $45 \mathrm{~Gy}$. Although there was initial response, the patient did not complete therapy and was lost to follow-up.

\section{Case 4}

A 35 year old Chinese female was admitted for excision of a breast lump of 5 months' duration. On examination, a $2.5-\mathrm{cm}$ lump which was firm and freely mobile was noted in the left breast. There was no axillary lymphadenopathy. Histological examination of the breast lump showed the presence of malignant melanoma. Search for a primary revealed a $3-\mathrm{cm}$ mass in the anal canal, which was excised. Histological examination confirmed the diagnosis of anorectal melanoma. The patient died 9 months later from extensive metastatic disease.

\section{Discussion}

Primary anorectal melanoma is a rare disease comprising less than $1 \%$ of all malignant tumours of the anus and rectum. ${ }^{5}$ Although it represents less than $1 \%$ of melanomas, it is the third most common primary site after the skin and retina. ${ }^{8}$ The tumour is thought to arise from neuroectodermal melanocytes in the anal mucosa and spread proximally into the rectal submucosa and lamina propria. Whether melanomas arise in the rectum itself is a subject of great controversy. ${ }^{1}$

Initial reports of the disease in predominantly Caucasian patients have been followed by increasing numbers of reported cases in non-whites. ${ }^{5-7}$ It may be that with increasing awareness and diagnostic skills a wider geographical distribution of the disease is becoming apparent. The authors believe that cases 1 and 3 represent the first reports of anorectal melanoma in Malay patients.

Late diagnosis of the disease at an advanced stage is common. ${ }^{9}$ Anorectal melanomas are often mistaken for benign anal conditions, as exemplified in case 1. The marked variations in gross appearance and absence of pigmentation in the amelanotic variety contribute to difficulties in diagnosis. Symptomatology is often typical of anorectal pathology. ${ }^{1,10}$ Case 4 represents an unusual presentation of anorectal melanoma since the metastatic lesion in the breast preceded the presentation of the primary lesion. This has not been reported in earlier series. ${ }^{1,5,9,10}$

Prognosis is related to stage of the disease ${ }^{1,7}$ and this is possibly best assessed by measuring tumour thickness as described by Breslow and the use of a modified Dukes' classification. ${ }^{3}$ In a series from the Memorial Sloan-Kettering Cancer Center, no patient with anal melanoma thicker than $1.7 \mathrm{~mm}$ survived 5 years. ${ }^{1}$ All four cases in this series presented with gross mass lesions, with metastatic lesions.

The treatment of anorectal melanoma is by surgery. The optimal extent of resection has not been determined. ${ }^{3,8}$ Reports have correctly emphasized that prognosis is influenced by the stage of the disease rather than on the type of operative pro-co cedure instituted.5,7 Nevertheless, surgical extirpation of the primary lesion should be carried out to prevent obstruction from occurring and in the hope of attaining an occasional cure. Abdominoperineal resection combined with prophylactic pelvic and inguinal lymph node dissection as advocated by Pack and Oropeza ${ }^{12}$ has fallen into disrepute because of the attendant complications and disappointing results. $3,5,10,11$

Controversy exists between local excision and abdominoperineal resection (APR) as the choice procedure. Quan et al. and Cooper et al. have suggested that local excision encompassing the entire tumour is as effective as radical resection. 13,14 Proponents of APR have illustrated higher local recurrence rates with the lesser procedure and that all 5-year survivors had the most radical operation. ${ }^{1,8,10}$ When inguinal lymph nodes are involved, therapeutic lymphadenectomy is advised. ${ }^{3,7}$

The currently available therapeutic agents are ineffective in the treatment of melanoma. ${ }^{5,7,15}$ Radiotherapy has also not resulted in any significant improvement in survival ${ }^{5}$ although there have been reports on its usage for palliation. ${ }^{8}$ 


\section{References}

1. Wanebo, H.J., Woodruff, J.M., Gist, H.F. \& Quan, S.H. Anorectal melanoma. Cancer 1981, 47: 1891-1900.

2. Moore, W.D. Recurrent melanosis of the rectum after previous removal from the verge of the anus in a man aged sixty-five. Lancet 1857, i: 290.

3. Adams, V.G. \& Efron, G. Current concepts and controversies concerning the etiology, pathogenesis, diagnosis and treatment of malignant tumours of the anus. Surgery 1987, 101: 253-266.

4. Quinn, D. \& Selah, C. Malignant melanoma of the anus in a Negro: report of a case and review of the literature. Dis Colon Rectum 1977, 20: 627-631.

5. Siegel, B., Cohen, D. \& Jacob, E.T. Surgical treatment of anorectal melanomas. Am J Surg 1983, 146: 336-338.

6. Chulani, H.I. Anal malignant melanoma: report of a case. Dis Colon Rectum 1977, 20: 517-520.

7. Boey, J., Chu, T.K., Wong, J. \& Ong, G.B. The surgical management of anorectal melanoma. Aust NZ J Surg 1981, 51: 132-136.

8. Angearas, U., Jonsson, N. \& Jonsson, P. Primary anorectal malignant melanoma. J Surg Oncol 1983, 22: $261-264$.
9. Abbas, J.S., Karakousis, C.P. \& Holyoake, E.D. Anorectal melanoma: clinical features, recurrence and patient survival. Int Surg 1980, 65: 423-426.

10. Chiu, Y.S., Krishnan, K.U. \& Beart, R.W. Malignant melanoma of the anorectum. Dis Colon Rectum 1980 , 23: $122-124$.

11. Stearns, Jr. M.W., Urmacher, G., Sternberg, S.S., Woodruff, J. \& Attiyeh, F. Cancer of the anal canal. Curr Probl Cancer 1980, 4: 1-44.

12. Pack, G.T. \& Oropeza, R. A comparative study of melanoma and epidermoid carcinoma of the anal canal. A review of 20 melanomas and 29 epidermoid carcinomas. Dis Colon Rectum 1967, 10: 161-176.

13. Quan, S.H., White, J.E. \& Reddish, M.R. Malignant melanoma of the anorectum. Dis Colon Rectum 1959, 2: 275-283.

14. Cooper, P.H., Mills, S.E. \& Allen, Jr. M.S. Malignant melanomas of the anus. Report of 12 cases and analyses of 255 additional cases. Dis Colon Rectum 1982, 25: 693-703.

15. Quan, S.H. Anal and para-anal tumours. Surg Clin North Am 1978, 58: 591-604. 